\title{
Generating Function and a Rodrigues Formula for the Polynomials in $d$-Dimensional Semiclassical Wave Packets
}

\author{
George A. Hagedorn* \\ Department of Mathematics and \\ Center for Statistical Mechanics and Mathematical Physics \\ Virginia Polytechnic Institute and State University \\ Blacksburg, Virginia 24061-0123, U.S.A. \\ E-mail: hagedorn@math.vt.edu
}

August 11, 2015

\begin{abstract}
We present a simple formula for the generating function for the polynomials in the $d$-dimensional semiclassical wave packets. We then use this formula to prove the associated Rodrigues formula.
\end{abstract}

\section{Introduction}

The generating function for 1-dimensional semiclassical wave packets is presented in formula (2.47) of [2]. In this paper, we present and prove the $d$-dimensional analog. As an application of this formula, we also prove the associated (multi-dimensional) Rodrigues formula.

These results have also been proven from a completely different point of view by Helge Dietert, Johannes Keller, and Stephanie Troppmann. See Lemma 3 and Section 3 (particularly Proposition 16) and formula (13) of [1]. See also [3]. We have also received a conjecture

*Partially Supported by National Science Foundation Grant DMS-1210982. 
from Tomoki Ohsawa [4] that the generating function result could be proved abstractly by using the formula for products of Hermite polynomials and the action of the metaplectic group.

Acknowledgements It is a pleasure to thank Raoul Bourquin and Vasile Gradinaru for motivating this work. It is also a pleasure to thank Johannes Keller, Tomoki Ohsawa, Sam Robinson, and Leonardo Mihalcea for their enthusiasm and numerous comments. It is also a pleasure to thank the referee whose comments improved this paper significantly. The author was supported in part by National Science Foundation grant DMS-1210982.

\section{Semiclassical Wave Packets}

The semiclassical wave packets depend on two invertible $d \times d$ complex matrices $A$ and $B$ that are always assumed to satisfy

$$
A^{*} B+B^{*} A=2 I \quad \text { and } \quad A^{t} B-B^{t} A=0 .
$$

They also depend on a phase space point $(a, \eta)$ that plays no role in the present work. After chosing a branch of the square root, we define

$$
\begin{aligned}
\varphi_{0}(A, B, \hbar, a, \eta, x)= & \pi^{-d / 4} \hbar^{-d / 4}(\operatorname{det} A)^{-1 / 2} \\
& \times \exp \left(-\frac{\left\langle(x-a), B A^{-1}(x-a)\right\rangle}{2 \hbar}+i \frac{\langle\eta,(x-a)\rangle}{\hbar}\right) .
\end{aligned}
$$

Here, and for the rest of this paper, we regard $\mathbb{R}^{d}$ as being embedded in $\mathbb{C}^{d}$, and for any two vectors $a \in \mathbb{C}^{d}$ and $b \in \mathbb{C}^{d}$, we use the notation

$$
\langle a, b\rangle=\sum_{j=1}^{d} \overline{a_{j}} b_{j} .
$$

For $1 \leq l \leq d$, we define the $l^{\text {th }}$ raising operator

$$
\mathcal{R}_{l}=\mathcal{A}_{l}(A, B, \hbar, 0,0)^{*}=\frac{1}{\sqrt{2 \hbar}}\left(\left\langle B e_{l},(x-a)\right\rangle-i\left\langle A e_{l},(-i \hbar \nabla-\eta)\right\rangle\right) .
$$

Then recursively, for any multi-index $k$, we define

$$
\varphi_{k+e_{l}}(A, B, \hbar, a, \eta, x)=\frac{1}{\sqrt{k_{l}+1}} \mathcal{R}_{l}\left(\varphi_{k}(A, B, \hbar, a, \eta)\right)(x) .
$$


For fixed $A, B, \hbar, a, \eta$, these wave packets form an orthonormal basis indexed by $k$. It is easy to see that

$$
\varphi_{k}(A, B, \hbar, a, \eta, x)=2^{-|k| / 2}(k !)^{-1 / 2} P_{k}(A, \hbar,(x-a)) \varphi_{0}(A, B, \hbar, a, \eta, x)
$$

where $P_{k}(A, \hbar,(x-a))$ is a polynomial of degree $|k|$ in $(x-a)$, although from this definition, it is not immediately obvious that $P_{k}(A, \hbar,(x-a))$ is independent of $B$.

Since they play no interesting role in what we are doing here, we henceforth assume $a=0$ and $\eta=0$.

\section{The Generating Function}

Our main result for the generating function is the following:

Theorem 3.1 The generating function for the family of polynomials $P_{k}(A, \hbar, x)$ is

$$
G(x, z)=\exp \left(-\left\langle\bar{z}, A^{-1} \bar{A} z\right\rangle+\frac{2}{\sqrt{\hbar}}\left\langle\bar{z}, A^{-1} x\right\rangle\right)
$$

I.e.,

$$
G(x, z)=\sum_{k} P_{k}(A, \hbar, x) \frac{z^{k}}{k !}
$$

Remark We make the unconventional definition $|A|=\sqrt{A A^{*}}$. By our conditions on the matrices $A$ and $B$, this forces $|A|$ to be real symmetric and strictly positive. We also have the polar decomposition $A=|A| U_{A}$, where $U_{A}$ is unitary. With this notation, we can write

$$
G(x, z)=\exp \left(-\left\langle U_{A} \bar{z}, \overline{U_{A}} z\right\rangle+\frac{2}{\sqrt{\hbar}}\left\langle U_{A} \bar{z},|A|^{-1} x\right\rangle\right)
$$

This equivalent formula is the one we shall actually prove.

We begin the proof of Theorem 3.1 with a lemma that provides an alternative formula for $\mathcal{R}_{l}$. From this formula and an induction on $|k|$, one can easily prove that $P_{k}(A, \hbar, x)$ is independent of $B$, because

$$
\overline{\varphi_{0}(A, B, \hbar, 0,0, x)} \varphi_{0}(A, B, \hbar, 0,0, x)=\pi^{-d / 2} \hbar^{-d / 2}|\operatorname{det} A|^{-1} \exp \left(-\frac{\left\langle x,|A|^{-2} x\right\rangle}{\hbar}\right) \text {. }
$$


Lemma 3.2 For any $\psi \in \mathcal{S}$,

$$
\left(R_{l} \psi\right)(x)=-\sqrt{\frac{\hbar}{2}} \frac{1}{\overline{\varphi_{0}(A, B, \hbar, 0,0, x)}}\left\langle A e_{l}, \nabla\left(\overline{\varphi_{0}(A, B, \hbar, 0,0, x)} \psi(x)\right)\right\rangle .
$$

Proof: The gradient on the right hand side of the equation in the lemma can act either on the $\overline{\varphi_{0}}$ or on the $\psi$. So, we get two terms when we compute this:

$$
\begin{aligned}
\sqrt{\frac{\hbar}{2}}\left(\frac { 1 } { 2 \hbar } \sum _ { j = 1 } ^ { d } \left\langleA e_{l},\left(e_{j}\left(\left\langle e_{j}, \bar{B}^{-1} x\right\rangle+\left\langle x, \bar{B}^{-1} e_{j}\right\rangle\right)\right\rangle \psi(x)\right.\right. \\
\left.-\left\langle A e_{l},(\nabla \psi)(x)\right\rangle\right) .
\end{aligned}
$$

The second term here is precisely the second term $\frac{1}{\sqrt{2 \hbar}}\left(-i\left\langle A e_{l},(-i \hbar \nabla) \psi(x)\right\rangle\right)$, in the expression for $\left(R_{l} \psi\right)(x)$. So, we need only show the first term here equals the first term, $\frac{1}{\sqrt{2 \hbar}}\left\langle B e_{l}, x\right\rangle \psi(x)$, in the expression for $\left(R_{l} \psi\right)(x)$.

To do this, we begin by noting that the first term here equals

$$
\begin{aligned}
& \frac{1}{2 \sqrt{2 \hbar}} \sum_{j=1}^{d}\left\langle A e_{l},\left(e_{j}\left(\left\langle e_{j}, \bar{B} \bar{A}^{-1} x\right\rangle+\left\langle x, \bar{B} \bar{A}^{-1} e_{j}\right\rangle\right)\right\rangle \psi(x)\right. \\
& =\frac{1}{2 \sqrt{2 \hbar}} \sum_{j=1}^{d}\left\langle A e_{l},\left(e_{j}\left(\left\langle e_{j}, \bar{B} \bar{A}^{-1} x\right\rangle+\overline{\left\langle\bar{B} \bar{A}^{-1} e_{j}, x\right\rangle}\right)\right\rangle \psi(x)\right. \\
& =\frac{1}{2 \sqrt{2 \hbar}} \sum_{j=1}^{d}\left\langle A e_{l},\left(e_{j}\left(\left\langle e_{j}, \bar{B} \bar{A}^{-1} x\right\rangle+\left\langle B A^{-1} e_{j}, x\right\rangle\right)\right\rangle \psi(x)\right. \\
& =\frac{1}{2 \sqrt{2 \hbar}} \sum_{j=1}^{d}\left\langle A e_{l},\left(e_{j}\left(\left\langle e_{j}, \bar{B} \bar{A}^{-1} x\right\rangle+\left\langle e_{j},\left(A^{-1}\right)^{*} B^{*} x\right\rangle\right)\right\rangle \psi(x)\right. \\
& =\frac{1}{\sqrt{2 \hbar}}\left\langle A e_{l}, \frac{\bar{B} \bar{A}^{-1}+\left(A^{-1}\right)^{*} B^{*}}{2} x\right\rangle \psi(x)
\end{aligned}
$$

Because of the relations satisfied by $A$ and $B, B A^{-1}$ is (real symmetric) $+i$ (real symmetric). So, its conjugate, $\bar{B} \bar{A}^{-1}$ has this same form. Thus, $\bar{B} \bar{A}^{-1}$ equals its transpose, which 
is $\left(A^{-1}\right)^{*} B^{*}$. So, the quantity of interest here equals

$$
\begin{aligned}
& \frac{1}{\sqrt{2 \hbar}}\left\langle A e_{l},\left(A^{-1}\right)^{*} B^{*} x\right\rangle \psi(x) \\
= & \frac{1}{\sqrt{2 \hbar}}\left\langle e_{l}, A^{*}\left(A^{-1}\right)^{*} B^{*} x\right\rangle \psi(x) \\
= & \frac{1}{\sqrt{2 \hbar}}\left\langle e_{l}, B^{*} x\right\rangle \psi(x) \\
= & \frac{1}{\sqrt{2 \hbar}}\left\langle B e_{l}, x\right\rangle \psi(x),
\end{aligned}
$$

which is what we had to show.

Proof of Theorem 3.1: By an induction on $|k|$, we prove there is a $|k|^{\text {th }}$ order polynomial $p_{k}$ in $d$ variables with the following properties:

- $\quad P_{k}(A, \hbar, x)=p_{k}\left(|A|^{-1} x / \sqrt{\hbar}\right)$.

$$
\text { - }\left(\frac{\partial}{\partial z}\right)^{k} G(x, z)=p_{k}\left(|A|^{-1} x / \sqrt{\hbar}-\overline{U_{A}} z\right) G(x, z) \text {. }
$$

The result then follows by setting $z=0$. We never compute the polynomial $p_{k}$ because it may be complicated. We also use the notation $p_{k}(A, \hbar, x)=p_{k}\left(\left(|A|^{-1} x / \sqrt{\hbar}\right)\right.$.

We define $p_{0}=1$. Below, we inductively compute $\left(\frac{\partial}{\partial z}\right)^{k+e_{l}} G(x, z)$. For our second condition above to hold, $p_{k+e_{l}}$ must be defined via the sum of formulas (3.1) and (3.2). This uniquely defines the polynomial $p_{k+e_{l}}$.

For $k=0$, the result is trivial since $P_{0}(A, \hbar, x)=1$.

For the induction step, it is sufficient to do the following for an arbitrary positive integer $l \leq d:$

Assuming we have already proved these for some $k$, we prove them for the multi-index $k+e_{l}$.

To do this, we begin by noting that

$$
\varphi_{k}(A, B, \hbar, 0,0, x)=\frac{1}{\sqrt{k !}} \mathcal{R}^{k}\left(\varphi_{0}(A, B, \hbar, 0,0)\right)(x) .
$$


Also,

$$
\varphi_{k}(A, B, \hbar, 0,0, x)=2^{-|k| / 2}(k !)^{-1 / 2} P_{k}(A, \hbar, x) \varphi_{0}(A, B, \hbar, 0,0, x) .
$$

So,

$$
\mathcal{R}^{k}\left(\varphi_{0}(A, B, \hbar, 0,0)\right)(x)=2^{-|k| / 2} P_{k}(A, \hbar, x) \varphi_{0}(A, B, \hbar, 0,0, x) .
$$

Thus, when we apply the $l^{\text {th }}$ raising operator, the polynomial $P_{k}(A, \hbar, x)$ gets changed to $\frac{1}{\sqrt{2}} P_{k+e_{l}}(A, \hbar, x)$.

Assuming the induction hypothesis, when we differentiate $\frac{\partial^{k} G}{\partial z^{k}}$ with respect to $z_{l}$, the $z_{l}$ derivative can act on the $G(x, z)$ or it can act on the $p_{k}\left(|A|^{-1} x / \sqrt{\hbar}-U_{A} z\right)$. When it acts on the $G(x, z)$, we obtain

$$
2\left\langle U_{A} e_{l},\left(|A|^{-1} x / \sqrt{\hbar}-\overline{U_{A}} z\right)\right\rangle p_{k}(A, \hbar, x) G(x, z) .
$$

From the induction hypotheses, this is a polynomial of degree $|k|+1$ evaluated at the argument $|A|^{-1} x / \sqrt{\hbar}-\overline{U_{A}} z$. Note that this result depends on the following calculation, with $G(x, z)$ written with the polar decomposition of $A$ :

$$
\begin{aligned}
\frac{\partial G}{\partial z_{l}}(x, z) & =\left(-\left\langle U_{A} e_{l}, \overline{U_{A}} z\right\rangle-\left\langle U_{A} \bar{z}, \overline{U_{A}} e_{l}\right\rangle+\frac{2}{\sqrt{\hbar}}\left\langle U_{A} e_{l},|A|^{-1} x\right\rangle\right) G(x, z) \\
& =2\left\langle U_{A} e_{l},\left(|A|^{-1} x / \sqrt{\hbar}-\overline{U_{A}} z\right)\right\rangle G(x, z) .
\end{aligned}
$$

When the $\frac{\partial}{\partial z_{l}}$ acts on the polynomial, we either get zero or a polynomial of degree $|k|-1$.

$$
\begin{aligned}
& -\left\langle\overline{\left(\nabla p_{k}\right)\left(|A|^{-1} x / \sqrt{\hbar}-\overline{U_{A}} z\right)}, \overline{U_{A}} e_{l}\right\rangle G(x, z) \\
= & -\left\langle U_{A} e_{l},\left(\nabla p_{k}\right)\left(|A|^{-1} x / \sqrt{\hbar}-\overline{U_{A}} z\right)\right\rangle G(x, z) .
\end{aligned}
$$

Recall that

$$
\left(R_{l} \psi\right)(x)=-\sqrt{\frac{\hbar}{2}} \frac{1}{\overline{\varphi_{0}(A, B, \hbar, 0,0, x)}}\left\langle A e_{l}, \nabla\left(\overline{\varphi_{0}(A, B, \hbar, 0,0, x)} \psi(x)\right)\right\rangle
$$


and that from our induction hypothesis,

$$
\begin{aligned}
& \overline{\varphi_{0}(A, B, \hbar, 0,0, x)} \varphi_{k}(A, B, \hbar, 0,0, x) \\
= & \pi^{-d / 2} \hbar^{-d / 2} 2^{-|k| / 2}(k !)^{-1 / 2}|\operatorname{det} A|^{-1} p_{k}(A, \hbar, x) \exp \left\{-\frac{\left\langle x,|A|^{-2} x\right\rangle}{\hbar}\right\} .
\end{aligned}
$$

When computing $\mathcal{R}_{l} \varphi_{k}$ by this formula, the gradient in $\mathcal{R}_{l}$ can act on the exponential or the $p_{k}(A, \hbar, x)$. When it acts on the exponential, we get

$$
\begin{aligned}
& 2^{-|k| / 2}(k !)^{-1 / 2} p_{k}(A, \hbar, x) \sqrt{\frac{2}{\hbar}}\left\langle A e_{l},|A|^{-2} x\right\rangle \varphi_{0}(A, B, \hbar, 0,0, x) \\
= & 2^{-(|k|+1) / 2} \\
& \sqrt{k_{l}+1}\left(\left(k+e_{l}\right) !\right)^{-1 / 2} \\
& \times 2\left\langle U_{A} e_{l},|A|^{-1} x / \sqrt{\hbar}\right\rangle p_{k}(A, \hbar, x) \varphi_{0}(A, B, \hbar, 0,0, x) .
\end{aligned}
$$

When the gradient in $\mathcal{R}_{l}$ acts on the $p_{k}(A, \hbar, x)$, in $\mathcal{R}_{l} \varphi_{k}$, we get the term

$$
\begin{gathered}
-\sqrt{\frac{\hbar}{2}} 2^{-|k| / 2}(k !)^{-1 / 2}\left\langle A e_{l}, \nabla_{x}\left(p_{k}(A, \hbar, x)\right)\right\rangle \varphi_{0}(A, B, \hbar, 0,0, x) \\
=-2^{-(|k|+1) / 2}(k !)^{-1 / 2}\left\langle A e_{l}, \sum_{j=1}^{d}\left\langle e_{j},\left(\nabla p_{k}\right)(A, \hbar, x)\right\rangle|A|^{-1} e_{j}\right\rangle \varphi_{0}(A, B, \hbar, 0,0, x) \\
=-2^{-(|k|+1) / 2}(k !)^{-1 / 2}\left\langle A e_{l},|A|^{-1}\left(\nabla p_{k}\right)(A, \hbar, x)\right\rangle \varphi_{0}(A, B, \hbar, 0,0, x) \\
=-2^{-(|k|+1) / 2} \sqrt{k_{l}+1}\left(\left(k+e_{l}\right) !\right)^{-1 / 2} \\
\times\left\langle U_{A} e_{l},\left(\nabla p_{k}\right)(A, \hbar, x)\right\rangle \varphi_{0}(A, B, \hbar, 0,0, x) .
\end{gathered}
$$

From (3.1) and (3.2) with $z=0$, we obtain

$$
2\left\langle U_{A} e_{l},|A|^{-1} x / \sqrt{\hbar}\right\rangle p_{k}(A, \hbar, x)-\left\langle U_{A} e_{l},\left(\nabla p_{k}\right)\left(|A|^{-1} x / \sqrt{\hbar}\right)\right\rangle .
$$

From (3.3) and (3.4) and taking into account the factor of $\sqrt{k_{l}+1}$ in $\mathcal{R}_{l}\left(\varphi_{k}\right)=\sqrt{k_{l}+1} \varphi_{k+e_{l}}$, we obtain

$$
\begin{aligned}
& P_{k+e_{l}}(A, \hbar, x) \\
= & 2\left\langle U_{A} e_{l},|A|^{-1} x / \sqrt{\hbar}\right\rangle p_{k}(A, \hbar, x)-\left\langle U_{A} e_{l},\left(\nabla p_{k}\right)\left(|A|^{-1} x / \sqrt{\hbar}\right)\right\rangle .
\end{aligned}
$$


The quantities of interest contain the same polynomial evaluated at the appropriate arguments, and $P_{k+e_{l}}(A, \hbar, x)=p_{k+e_{l}}(A, \hbar, x)$. Since $l$ is arbitrary, with $1 \leq l \leq d$, the result is true for all multi-indices with order $|k|+1$, and the induction can proceed.

\section{The Rodrigues Formula}

As an application of the Generating Function formula, we prove a multi-dimensional Rodrigues formula for these polynomials. The result is

Theorem 4.1 In d-dimensions, with the convention that $|A|$ is the positive square root of $A A^{*}$,

$$
P_{k}(A, \hbar, x)=\exp \left(\frac{\left\||A|^{-1} x\right\|^{2}}{\hbar}\right)\left(-\sqrt{\hbar} A^{*} \nabla_{x}\right)^{k} \exp \left(-\frac{\left\||A|^{-1} x\right\|^{2}}{\hbar}\right) .
$$

Remark By scaling, it is sufficient to prove this for $\hbar=1$.

We begin the proof of this result with a lemma that embodies a special case of the chain rule for high order derivatives in $d$-dimensions.

Lemma 4.2 Assume $F: \mathbb{R}^{d} \rightarrow \mathbb{R}^{d}$ is $C^{m}$ and that $M: \mathbb{R}^{d} \rightarrow \mathbb{R}^{d}$ is linear. Then viewing gradients as column vectors and using multi-index notation,

$$
\left(\nabla_{x}\right)^{m} F(M x)=\left(\left(M^{t} \nabla_{y}\right)^{m} F\right)_{y=M x}
$$

One proves this by induction on $|m|$, proving that each component is correct.

Proof of Theorem 4.1 Using this technical result and the generating function,

$$
\begin{aligned}
& P_{k}(A, x) \\
= & \left(\nabla_{z}\right)^{k} \exp \left(-\left(\overline{U_{A}} z\right)^{t}\left(\overline{U_{A}} z\right)+2\left(\overline{U_{A}} z\right)^{t}\left(|A|^{-1} x\right)\right)_{z=0} \\
= & \left(\nabla_{z}\right)^{k} \exp \left(-\left(\overline{U_{A}} z-|A|^{-1} x\right)^{t}\left(\overline{U_{A}} z-|A|^{-1} x\right)\right) \exp \left(\left(|A|^{-1} x\right)^{t}\left(|A|^{-1} x\right)\right)_{z=0} \\
= & \exp \left(\left(|A|^{-1} x\right)^{t}\left(|A|^{-1} x\right)\right)\left(\nabla_{z}\right)^{k} \exp \left(-\left(\overline{U_{A}} z-|A|^{-1} x\right)^{t}\left(\overline{U_{A}} z-|A|^{-1} x\right)\right)_{z=0}
\end{aligned}
$$




$$
\begin{aligned}
& =\exp \left(\left(|A|^{-1} x\right)^{t}\left(|A|^{-1} x\right)\right)\left(\left(\overline{U_{A}}\right)^{t} \nabla_{w}\right)^{k} \exp \left(-\left(w-|A|^{-1} x\right)^{t}\left(w-|A|^{-1} x\right)\right)_{w=0} \\
& =\exp \left(\left(|A|^{-1} x\right)^{t}\left(|A|^{-1} x\right)\right)\left(\left(\overline{U_{A}}\right)^{t} \nabla_{w}\right)^{k} \exp \left(-(w-u)^{t}(w-u)\right)_{\substack{w=0 \\
u=|A|^{-1} x}} \\
& =\exp \left(\left(|A|^{-1} x\right)^{t}\left(|A|^{-1} x\right)\right)\left(-\left(\overline{U_{A}}\right)^{t} \nabla_{u}\right)^{k} \exp \left(-(w-u)^{t}(w-u)\right)_{\substack{w=0 \\
u=|A|^{-1} x}} \\
& =\exp \left(\left(|A|^{-1} x\right)^{t}\left(|A|^{-1} x\right)\right)\left(-\left(\overline{U_{A}}\right)^{t}|A|^{t} \nabla_{x}\right)^{k} \exp \left(-\left(w-|A|^{-1} x\right)^{t}\left(w-|A|^{-1} x\right)\right)_{w=0} \\
& \left.=\exp \left(\left(|A|^{-1} x\right)^{t}\left(|A|^{-1} x\right)\right)\left(-\left(\overline{U_{A}}\right)^{t}|A|^{t} \nabla_{x}\right)^{k} \exp \left(-|A|^{-1} x\right)^{t}\left(|A|^{-1} x\right)\right) .
\end{aligned}
$$

However, $|A|^{t}=|A|$ is real, and with our unusual convention, $A=|A| U_{A}$. So, $\left(\overline{U_{A}}\right)^{t}|A|=A^{*}$. This proves the theorem.

\section{References}

[1] Dietert, H., Keller, J., and Troppmann, S.: An Invariant Class of Hermite Type Multivariate Polynomials for the Wigner Transform. (2015 preprint, arXiv:1505.06192).

[2] Hagedorn, G.A.: Raising and Lowering Operators for Semiclassical Wave Packets. Ann. Phys. 269, 77-104 (1998).

[3] Keller, J.F.: Quantum Dynamics on Potential Energy Surfaces. Doctoral Thesis, Technische Universität - München (2015).

[4] Ohsawa, T.: private communication (2015). 